\title{
Symmetry of the charge density wave in cuprates
}

\author{
Ashot Melikyan ${ }^{1,2}$ and M. R. Norman 1 , * \\ ${ }^{1}$ Materials Science Division, Argonne National Laboratory, Argonne, IL, 60439 \\ ${ }^{2}$ American Physical Society, Ridge, NY 11961
}

(Dated: May 8, 2019)

\begin{abstract}
We derive and analyze an effective Ginzburg-Landau (GL) functional for a charge density wave (CDW) for a model of electrons on a tight binding square lattice with density-density interactions. We show, using realistic electronic dispersions for the cuprates, that for the simplest GL theory, the preferred symmetry is typically uni-directional (stripe) type, but inclusion of third-order terms tends to destabilize this in favor of a checkerboard pattern depending on the strength and range of the interaction. This is of interest given the recent observation of such charge order in underdoped $\mathrm{YBa}_{2} \mathrm{Cu}_{3} \mathrm{O}_{6+x}$.
\end{abstract}

PACS numbers: 71.45.Lr, 74.20.De, 74.72.-h

\section{INTRODUCTION}

Modulations of the local density of states in cuprates, which were observed by scanning tunneling microscopy in $\mathrm{Bi}_{2} \mathrm{Sr}_{2} \mathrm{CaCu}_{2} \mathrm{O}_{8+\delta}(\mathrm{Bi} 2212)^{1-6}$ and $\mathrm{Ca}_{2-x} \mathrm{Na}_{x} \mathrm{CuO}_{2} \mathrm{Cl}_{2}{ }^{7,8}$, have attracted significant attention. Although a number of theories for these observations have been proposed, ${ }^{9-19}$ the nature of the modulated state is still debated. The modulations are strongest in the underdoped region of the phase diagram, a faithful description of which could be a difficult task. Fluctuations of the superconducting order parameter, intrinsic disorder, and competing/coexisting order, all potentially play a role in underdoped samples.

Nevertheless, short of knowing the exact Hamiltonian governing the low energy phenomenology of the cuprates, it is instructive to isolate the role of separate contributions by focusing on one of them at a time. The goal of this article is to study the influence of the electronic dispersion in determining the nature of potential charge density waves in cuprates. This is realized by deriving a Ginzburg-Landau (GL) free energy and analyzing the symmetry of the possible charge modulations, with the coefficients of the free energy determined from dispersions extracted from angle-resolved photoemission data.

\section{GINZBURG-LANDAU FREE ENERGY FOR A CDW}

Our starting point is the generalized extended Hubbard model of interacting electrons on a tight binding square lattice. In terms of electron creation operators $\psi_{\mathbf{k}, \sigma}^{\dagger}$, the Hamiltonian of the model is:

$$
H=\sum_{\mathbf{k}, \sigma}\left(\xi_{\mathbf{k}}-\mu\right) \psi_{\mathbf{k} \sigma}^{\dagger} \psi_{\mathbf{k} \sigma}+\frac{1}{2} \sum_{\mathbf{r}, \mathbf{r}^{\prime}} g\left(\mathbf{r}-\mathbf{r}^{\prime}\right) n(\mathbf{r}) n\left(\mathbf{r}^{\prime}\right)
$$

where $\xi_{\mathbf{k}}$ denotes the energy dispersion (with $\mu$ the chemical potential), $\mathbf{r}$ and $\mathbf{r}^{\prime}$ are the sites of the lattice, and $n(\mathbf{r})$ is the charge density. The model reduces to the Hubbard model when $g\left(\mathbf{r}-\mathbf{r}^{\prime}\right)=g_{0} \delta_{\mathbf{r}, \mathbf{r}^{\prime}}$, and to the so-called

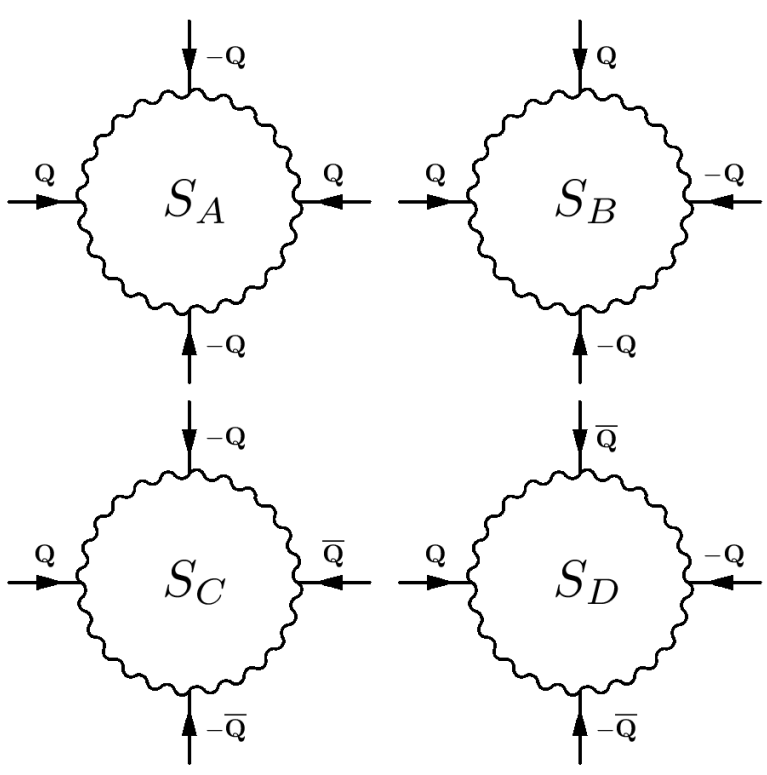

FIG. 1: Diagrams corresponding to the GL coefficients $S_{A}$, $S_{B}, S_{C}$, and $S_{D}$.

$U-V$ (extended Hubbard) model when $g\left(\mathbf{r}-\mathbf{r}^{\prime}\right)$ is also non zero for the nearest neighbor sites $\mathbf{r}^{\prime}=\mathbf{r} \pm a \hat{x}, \mathbf{r} \pm a \hat{y}$. As a function of the charge-density-wave order parameter $\Delta(\mathbf{Q})$, the effective GL free energy (per lattice site) to quartic order can be written as

$$
\begin{gathered}
F(\Delta)-F(0)=\sum_{\mathbf{q}, \Omega}\left|\Delta_{\mathbf{q}, \Omega}\right|^{2}\left(-\frac{1}{2 g_{\mathbf{q}}}-\chi(\mathbf{q}, \Omega)\right) \\
-\frac{2}{3} \sum_{q_{1}, q_{2}, q_{3}} \Delta_{q_{1}} \Delta_{q_{2}} \Delta_{q_{3}} \delta\left(\sum_{n=1}^{3} q_{n}\right) S_{3}\left(q_{1}, q_{2}, q_{3}\right) \\
+\frac{1}{2} \sum_{q_{1}, q_{2}, q_{3}, q_{4}} \Delta_{q_{1}} \Delta_{q_{2}} \Delta_{q_{3}} \Delta_{q_{4}} \delta\left(\sum_{n=1}^{4} q_{n}\right) S_{4}\left(q_{1}, q_{2}, q_{3}, q_{4}\right)
\end{gathered}
$$

where the summations are performed over the momenta $\mathbf{q}_{n}$ and the bosonic Matsubara frequencies $\Omega_{n}=2 \pi n / \beta$, 
$F(0)$ is the free energy of the normal state $\left(\Delta_{q}=0\right)$, and the notation $q_{n}=\left(\mathbf{q}_{n}, \Omega_{n}\right)$ is used for brevity. The coefficients of the expansion can be expressed through Greens functions $G_{\mathbf{k}, \omega_{n}}=\left(i \omega_{n}-\xi_{\mathbf{k}}+\mu\right)^{-1}$ as

$$
\begin{aligned}
\chi(\mathbf{q}, \Omega) & =-\frac{1}{N \beta} \sum_{k} G_{k} G_{k+q} \\
S_{3}\left(\mathbf{q}_{i}, \Omega_{i}\right) & =\frac{1}{N \beta} \sum_{k} G_{k} G_{k+q_{1}} G_{k+q_{1}+q_{2}} \\
S_{4}\left(\mathbf{q}_{i}, \Omega_{i}\right) & =\frac{1}{N \beta} \sum_{k} G_{k} G_{k+q_{1}} G_{k+q_{1}+q_{2}} G_{k+q_{1}+q_{2}+q_{3}}
\end{aligned}
$$

where $k=\left(\mathbf{k}, \omega_{n}\right)$. After summation over fermionic frequencies $\omega_{n}=\pi(2 n+1) / \beta$, one obtains the usual result for the (2D) charge susceptibility

$$
\chi(\mathbf{q}, \Omega)=-\int \frac{a^{2} d \mathbf{k}}{(2 \pi)^{2}} \frac{f\left(\xi_{\mathbf{k}}\right)-f\left(\xi_{\mathbf{k}+\mathbf{q}}\right)}{\xi_{\mathbf{k}}-\xi_{\mathbf{k}+\mathbf{q}}+i \Omega}
$$

with the integral over the first Brillouin zone. In our mean field theory, we only need the cubic and quartic coefficient functions $S_{3}$ and $S_{4}$ for $\Omega_{i}=0$

$S_{3}\left(\mathbf{p}_{i}\right)=\int \frac{a^{2} d \mathbf{k}}{(2 \pi)^{2}}\left[\frac{f\left(\xi_{\mathbf{k}}\right)}{\left(\xi_{\mathbf{k}}-\xi_{\mathbf{k}+\mathbf{p}_{1}}\right)\left(\xi_{\mathbf{k}}-\xi_{\mathbf{k}+\mathbf{p}_{1}+\mathbf{p}_{2}}\right)}+c . p.\right]$

where c.p. denotes the two other terms obtained by cyclic permutation of the momenta $\left(\mathbf{k}, \mathbf{k}+\mathbf{p}_{1}, \mathbf{k}+\mathbf{p}_{1}+\mathbf{p}_{2}\right)$. Similarly, for $S_{4}$ one finds

$$
\begin{aligned}
& S_{4}\left(\mathbf{p}_{i}\right)=\int \frac{a^{2} d \mathbf{k}}{(2 \pi)^{2}}\left(f\left(\xi_{\mathbf{k}}\right)\left(\xi_{\mathbf{k}}-\xi_{\mathbf{k}+\mathbf{p}_{1}}\right)^{-1}\right. \\
& \left.\left(\xi_{\mathbf{k}}-\xi_{\mathbf{k}+\mathbf{p}_{1}+\mathbf{p}_{2}}\right)^{-1}\left(\xi_{\mathbf{k}}-\xi_{\mathbf{k}+\mathbf{p}_{1}+\mathbf{p}_{2}+\mathbf{p}_{3}}\right)^{-1}+\text { c.p. }\right)
\end{aligned}
$$

where c.p. denotes the three other terms obtained by cyclic permutation of the momenta $\left(\mathbf{k}, \mathbf{k}+\mathbf{p}_{1}, \mathbf{k}+\mathbf{p}_{1}+\right.$ $\left.\mathbf{p}_{2}, \mathbf{k}+\mathbf{p}_{1}+\mathbf{p}_{2}+\mathbf{p}_{3}\right)$. As the temperature or the effective interaction $g\left(\mathbf{r}-\mathbf{r}^{\prime}\right)$ varies, for a specific set of equivalent momenta $\mathbf{Q}_{i}$, the quadratic coefficient of the GL expansion may change sign, and a phase transition takes place. In the vicinity of such a transition, it is sufficient to consider only the coefficients of the GL free energy evaluated at momenta $\mathbf{Q}_{i}$ and their harmonics. We will be specifically interested in situations where these equivalent momenta lie along the symmetry lines of the Brillouin zone, and thus the total number of them is restricted to four for the square lattice (typically, the maxima of $\chi$ will lie along such lines). Consider first a simplified expansion where only the fundamental harmonics at $\mathbf{Q}_{i}$ are retained. In such a case, the GL expansion reduces to

$$
\begin{aligned}
& F(\Delta)-F(0)=r_{\mathbf{Q}}\left(\left|\Delta_{\mathbf{Q}}\right|^{2}+\left|\Delta_{\overline{\mathbf{Q}}}\right|^{2}\right)+ \\
& \frac{1}{2}\left(2 S_{A}+4 S_{B}\right)\left(2 \gamma\left|\Delta_{\mathbf{Q}}\right|^{2}\left|\Delta_{\overline{\mathbf{Q}}}\right|^{2}+\left|\Delta_{\mathbf{Q}}\right|^{4}+\left|\Delta_{\overline{\mathbf{Q}}}\right|^{4}\right)
\end{aligned}
$$

where $\overline{\mathbf{Q}} \perp \mathbf{Q}$,

$$
r_{\mathbf{Q}}=-g_{\mathbf{Q}}^{-1}-2 \chi(\mathbf{Q}),
$$

$\Omega_{i}=0$ is implied, and the coefficients $S_{A, B, C, D}$, which are described by the diagrams in Fig. 1, are defined as

$$
\begin{aligned}
& S_{A}=S_{4}(\mathbf{Q},-\mathbf{Q}, \mathbf{Q},-\mathbf{Q}) \\
& S_{B}=S_{4}(\mathbf{Q}, \mathbf{Q},-\mathbf{Q},-\mathbf{Q}) \\
& S_{C}=S_{4}(\mathbf{Q},-\mathbf{Q}, \overline{\mathbf{Q}},-\overline{\mathbf{Q}}) \\
& S_{D}=S_{4}(\mathbf{Q}, \overline{\mathbf{Q}},-\mathbf{Q},-\overline{\mathbf{Q}})
\end{aligned}
$$

with $\gamma=\frac{8 S_{C}+4 S_{D}}{2 S_{A}+4 S_{B}}$. Formally identical GL expansions have been studied in the past, and for a momentumindependent interaction have been recently employed by Yao et al. in connection with CDWs that occur in the rare earth tri-tellurides (Ref. 20). The stability requirement dictates that $2 S_{A}+4 S_{B}>0$ and $\gamma>-1$. Provided these are satisfied, the symmetry of the CDW in the ordered phase is determined 18 by $\gamma$ : when $\gamma>1$, the free energy is minimized by choosing either $\Delta_{\mathbf{Q}}=0$ or $\Delta \overline{\mathbf{Q}}=0$ resulting in one-dimensional stripes. For $\gamma<1$, the minimum of the GL free energy is achieved by choosing $\left|\Delta_{\mathbf{Q}}\right|=\left|\Delta_{\overline{\mathbf{Q}}}\right|$, and the CDW has a two-dimensional "checkerboard" pattern.

\section{CHARGE SUSCEPTIBILITY AND INSTABILITY WAVE VECTORS IN CUPRATES}

The calculations were performed for three sets of sixparameter tight-binding fits to the dispersion, based on angle-resolved photoemission data, that were previously used to model the spin susceptibility ${ }^{21}$. The coefficients of these dispersions (tb1, tb2, and tb4) can be found in that work. tb1 is based on earlier photoemission data for Bi2212 22 and is characterized by a van Hove singularity at $(\pi, 0)$ that is $34 \mathrm{meV}$ below the Fermi energy, resulting in a sizable anisotropy of the Fermi velocity around the Fermi surface. tb2 is based on more recent photoemission data for Bi2212 23 which indicates an isotropic Fermi velocity. tb4 is based on photoemission data for underdoped $\mathrm{La}_{2-x} \mathrm{Sr}_{x} \mathrm{CuO}_{4}$ (LSCO) ${ }^{24}$.

In Fig. 2, we show $\chi(\mathbf{q}, \Omega=0)$. There are two sets of maxima. One set forms a box-like structure around the zone center, and it is this set which will be assumed to dominate the charge response since we anticipate that $g_{\mathbf{q}}$ will be maximal at $q=0$. The second set is concentrated around the $(\pi, \pi)$ point, and is thought to dominate the spin response since the superexchange interaction is maximal at this point. For the first set, the absolute maximum of $\chi$ typically occurs along the zone diagonal $\left(q_{x}=q_{y}\right)$. This can be qualitatively understood by employing an argument due to Schulz ${ }^{25}$. Although a bond aligned vector does a better job of nesting the antinodal sections of the Fermi surface near $(\pi, 0)$, a diagonal vector connects twice as many Fermi surface faces. Therefore, were it not for the momentum dependence of 

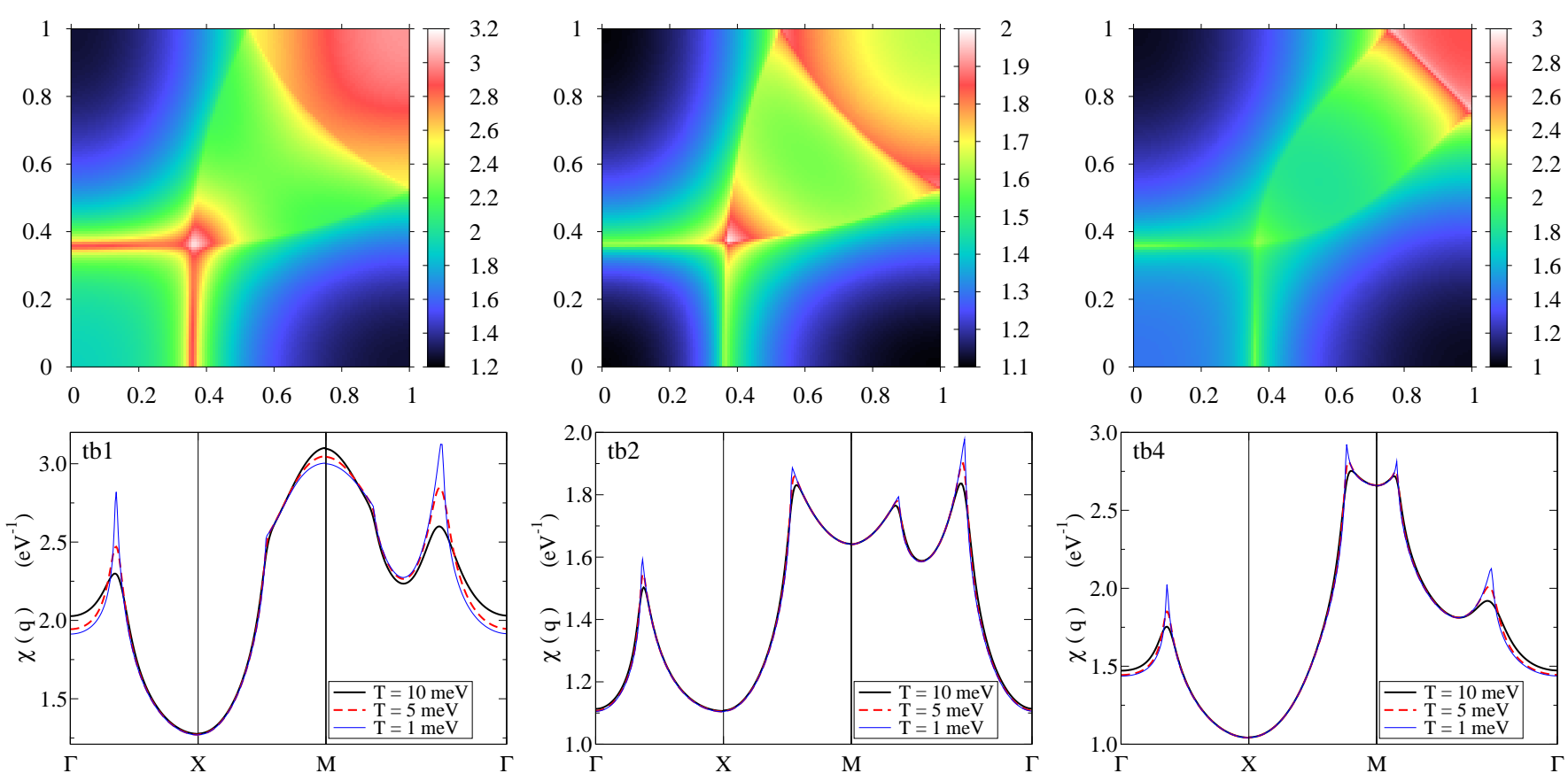

FIG. 2: (Color online) $\chi(\mathbf{q})$ calculated from the tight binding dispersions tb1, tb2, and tb4 (left to right) ${ }^{21}$. Top row: Twodimensional map of $\chi(\mathbf{q})$ for $T=0.001 t$, where $t$ is the near neighbor hopping parameter of the tight binding fit for $\xi_{\mathbf{k}}$. Since $\chi(\mathbf{q})$ is four-fold symmetric, only one quarter of the Brillouin zone is shown. Bottom row: $\chi(\mathbf{q})$ calculated for $T=1$ meV, 5 $\mathrm{meV}$, and $10 \mathrm{meV}$ along the various symmetry lines of the zone, with $\Gamma=(0,0), X=(1,0)$, and $M=(1,1)$ in $\pi / a$ units.

the interaction, one would conclude that the CDW typically cannot be directed along the bond directions, at least within the model considered here.

In two dimensions, the susceptibility does not diverge, even at $T=0$, unless the faces of the Fermi surfaces are perfectly nested. This is never the case for a realistic dispersion, although the Fermi surface does contain approximately nested sections that are responsible for the sharp peaks in $\chi$. This is illustrated in Fig. 3 for the peak lying along $\Gamma-X$. The discontinuities of the slope at momenta $\mathbf{q}_{1}$ and $\mathbf{q}_{2}$ (which define the plateau in the insets) are due to the points of the Fermi surface $\mathbf{k}$ and $\mathbf{k}^{\prime}$ separated by $\mathbf{q}_{i}$ such that the tangents to the Fermi surface at $\mathbf{k}$ and $\mathbf{k}^{\prime}$ are parallel. The lack of divergence in the susceptibility implies that the charge-density wave can develop only if the interaction strength exceeds a critical value.

The ordering vector in cuprates observed by scanning tunneling microscopy lies along the $\mathrm{Cu}-\mathrm{O}$ bonds, in contrast with our results for $\chi$. On the other hand, the true ordering vector is determined by the product of $\chi$ and $g$. We anticipate that $g_{\mathbf{q}}$ has a maximum at $q=0$ and falls off smoothly with increasing $q$. If this fall off is relatively isotropic and steep enough, then, as demon-

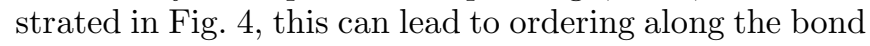
direction instead, since $q$ along the diagonal of the box structure shown in Fig. 2 is $\sqrt{2}$ times larger than $q$ along the bond direction. In general, the momentum $\mathbf{q}=\mathbf{Q}$ at which the charge-density instability occurs is determined
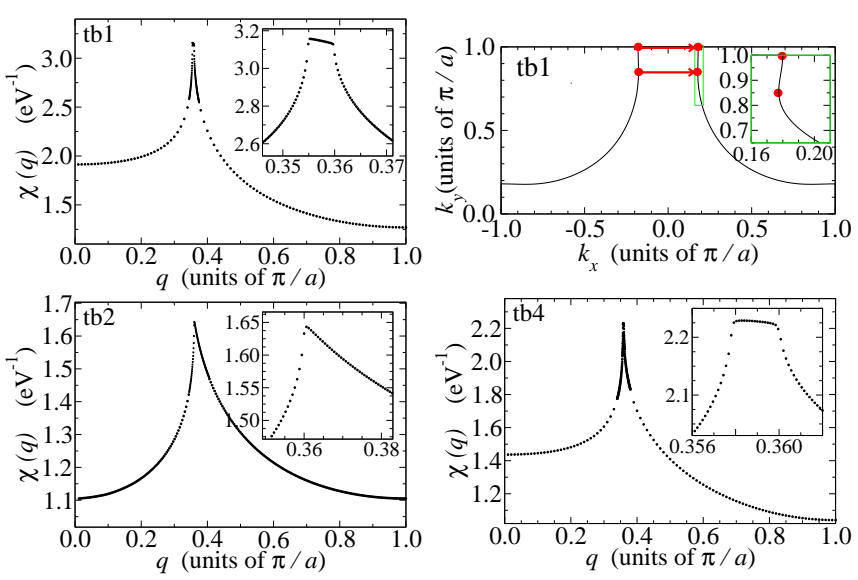

FIG. 3: (Color online) Susceptibility for $T=0$ along $\Gamma-X$ for dispersions tb1 (top left), tb2 (bottom left) and tb4 (bottom right). In all cases, $\chi$ is finite due to imperfect nesting. The Fermi surface for tb1 is shown in the top right panel. For dispersions tb1 and tb4, the susceptibility has two cusps, corresponding to momenta connecting points in a quadrant of the zone (shown for tb1 by the dots) with their partners in the adjacent quadrant. The characteristic bending of the Fermi surface near the antinodal direction, which is similar for the tb1 and tb4 fits, is absent for the tb2 dispersion where the susceptibility has a single cusp. In all cases, on one side of each cusp at $Q_{x 0}$, the slope of $\chi\left(Q_{x}, 0\right)$ is finite, while on the other side, $\chi\left(Q_{x 0}, 0\right)-\chi\left(Q_{x}, 0\right) \propto\left|Q_{x}-Q_{x 0}\right|^{1 / 2}$ and the slope is infinite. 
by the highest temperature such that the condition

$$
-\frac{1}{2 g_{\mathbf{q}}}=\chi\left(\mathbf{q}, T_{c}\right)
$$

is satisfied. For concreteness, we assume that the interaction has the following simplified form:

$$
-\frac{1}{2 g_{\mathbf{q}}} \approx \alpha+\beta|a \mathbf{q}|^{2}
$$

As this small $q$ expansion is most sensitive to the longer range part of $g$, our simple approximation cannot be directly mapped onto the extended Hubbard model mentioned in Section II.

Given this form of the interaction, the first task is to identify the momentum $\mathbf{Q}$ and the temperature $T_{c}$ at which the CDW first develops. As shown in Fig. 5, the solutions generally fall in four different classes. For a fixed curvature $\beta$, there are no solutions provided that $\alpha$ is large enough. This was to be expected, as the logarithmic divergence of the susceptibility is cut off due to imperfect nesting. Thus, for sufficiently weak interactions, no ordering occurs even at $T=0$. In the opposite limit of strong interactions and medium- to long-range interactions, the instability is at $q=0$. This is due to the strong reduction in the momentum dependence of $\chi$ as the temperature is raised, as can be appreciated from Fig. 4. In this high $T$ limit, the susceptibility maxima are confined to the region near the $M$ point of the zone, which leads to solutions for these wave vectors for small values of the curvature $\beta \lesssim 0.1$. This is not shown in Fig. 5. The reason is that the parabolic approximation we apply for $1 / g_{\mathbf{q}}$ is only valid near $q=0$. We anticipate that for larger values of $q$, the charge interaction is suppressed, and the magnetic interactions become dominant instead.

We therefore focus on the more pertinent cases of the two regions that are shown in Fig. 5 by the green circles and blue diamonds. In these cases, the instability occurs either at a momentum along $\Gamma-X$ (green circles) or $\Gamma-M$ (blue diamonds); two representative examples are shown in Fig. 4 (solid green and dashed blue parabolas). In either case, the ordering momentum lies in the immediate vicinity of the "box" structure that surrounds the $\Gamma$-point (Fig. 2). It is straightforward to show that the boundary separating the regions shown by the green circles and the gray "+" signs in the parameter space must be a straight line. This boundary corresponds to the limit $T_{c} \rightarrow 0$; in Fig. 4 it represents a family of parabolas with different $\alpha$ and $\beta$, all passing through the maximum of the zero-temperature susceptibility at a momentum $Q_{0} \approx 0.355 \pi / a$ (Fig. 3, tb1). The boundary is thus described by a straight line:

$$
\alpha+\beta a^{2} Q_{0}^{2}=\chi\left(T=0, Q_{0}\right)
$$

The other main features of the instability diagram can be understood in a similar fashion.

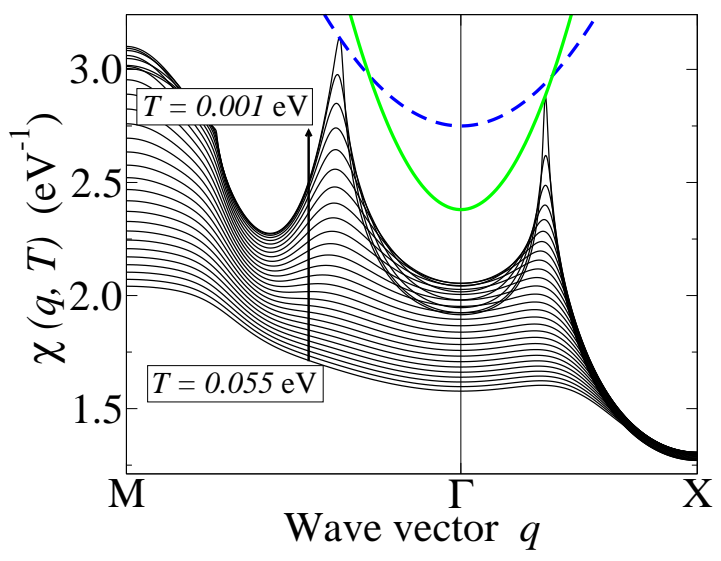

FIG. 4: (Color online) Depending on the momentum dependence of the interaction $g_{\mathbf{q}}$, a CDW with modulations along the $\mathrm{Cu}-\mathrm{O}$ bonds $(\Gamma-X)$ can be stabilized despite the fact that the maximum of the susceptibility along this direction is smaller than the peak value along $\Gamma-M$. Thin solid lines describe the momentum dependence of susceptibility for the tb1 dispersion along the contour $M-\Gamma-X$, for temperatures from $T=0.001 \mathrm{eV}$ to $T=0.055 \mathrm{eV}$, with increments of $T=0.002$ $\mathrm{eV}$. The inverse interaction strength $-1 / 2 g_{\mathbf{q}}=\alpha+\beta(a \mathbf{q})^{2}$ for two different sets of parameters $(\alpha, \beta)$ is shown by solid green $\left(\alpha=2.38 \mathrm{eV}^{-1}, \beta=0.40 \mathrm{eV}^{-1}\right)$ and dashed blue $(\alpha=2.75$ $\left.\mathrm{eV}^{-1}, \beta=0.15 \mathrm{eV}^{-1}\right)$ parabolas.

\section{QUARTIC TERMS AND THE SYMMETRY OF THE CDW IN CUPRATES}

Once the ordering momentum $\mathbf{Q}$ is known, the coefficients of the Ginzburg-Landau free energy, evaluated at the transition temperature $T=T_{c}$, can be computed rather easily. Fig. 6] illustrates the temperature dependence of the quartic coefficients calculated for the momentum $\mathbf{Q}=Q_{0} \hat{x}$, where $Q_{0} \approx 0.357 \pi / a$ is the instability momentum for a representative case of $\alpha=2.4 \mathrm{eV}^{-1}$ and $\beta=0.38 \mathrm{eV}^{-1}$. At high temperatures $(T \gg t)$, the leading-order result for all coefficients $S_{i}(i=A, B, C, D)$ has the same functional form $48 T^{-3}+\ldots$, as can be shown by a straightforward expansion of the integrand in Eq. (8) in powers of $\xi_{k} / T$. In the opposite limit of low temperatures, the coefficients $S_{A}, S_{C}$, and $S_{D}$ diverge as $T^{-3 / 2}$, while $S_{B}$ appears to remain finite. At high temperatures, $\gamma \rightarrow 2$, independent of any particular form of the dispersion. As can be seen from Fig. 6] $\gamma>1$ is satisfied at all temperatures. Therefore, in the simplified description where the additional harmonics are neglected, one would conclude that $1 \mathrm{D}$ stripe ordering at a momentum $Q_{0}$ is the preferred state. The effect of the harmonics will be discussed next.

Notice that while the coefficients of the quartic term in Eq. (9) are nominally determined only by the dispersion $\xi_{\mathbf{k}}$ (Eqs. (8) and (11)), they also indirectly depend on the interaction, $g_{\mathbf{q}}$, since this enters into the determination of the ordering vector as discussed above. However, the functional form of the interaction affects quartic coeffi- 


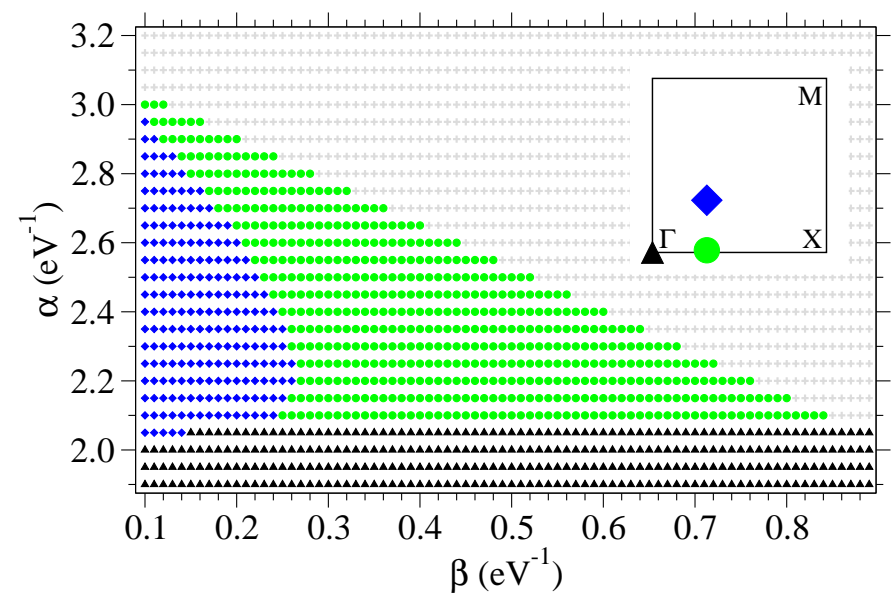

FIG. 5: (Color online) Instability diagram for the tb1 dispersion. The region marked by gray "+" symbols corresponds to interactions so weak that even at $T=0$, no CDW ordering occurs. For $(\alpha, \beta)$ in the parts of the diagram marked by green circles (blue diamonds), the instability happens at a momentum lying along $\Gamma-X(\Gamma-M)$. Even for moderately localized interactions ( $\beta$ small) the instability first appears at $\mathbf{q}=0$, provided that the interaction is sufficiently strong $\left(\alpha \lesssim 2 \mathrm{eV}^{-1}\right)$.

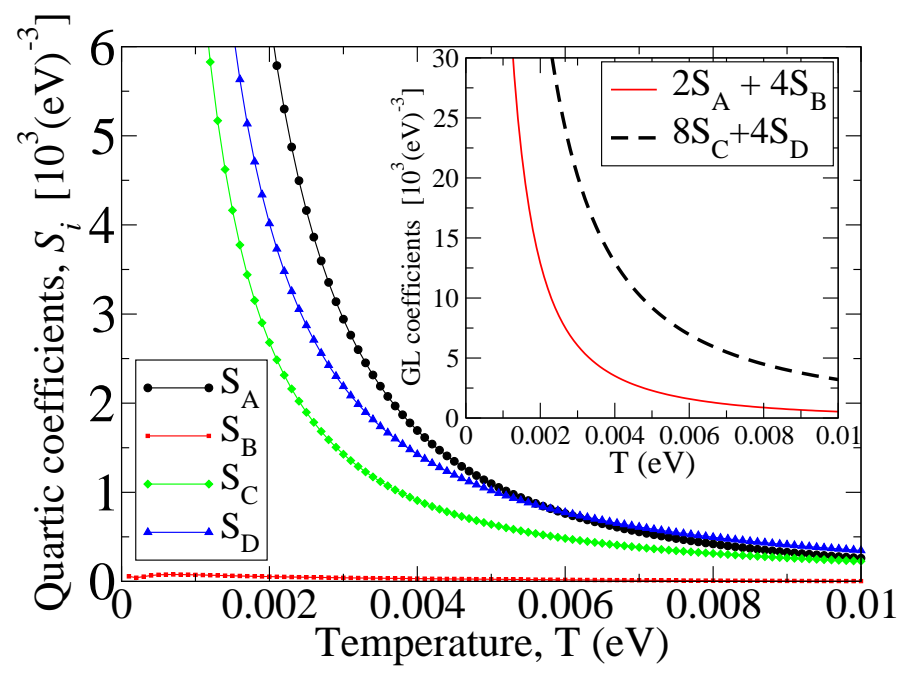

FIG. 6: (Color online) Temperature dependence of the quartic coefficients $S_{A}, S_{B}, S_{C}$, and $S_{D}$ for $\mathbf{Q} \approx(0.357 \pi / a, 0)$ (tb1 dispersion). The inset shows the linear combinations of the quartic coefficients that determine the symmetry of the charge density wave. The condition $\gamma=\left(8 S_{C}+4 S_{D}\right) /\left(2 S_{A}+4 S_{B}\right)>$ 1 , which is fulfilled for all temperatures in this case, corresponds to one-dimensional stripes.

cients in another fashion, which turns out to be rather pronounced. When deriving $F(\Delta)$, we left out of the final expression Eq. (9) all momenta but those where the instability first develops. It happens that the inclusion of the third-order terms in the effective action involves harmonics of the form $\Delta_{2 \mathbf{Q}}$ and $\Delta_{ \pm \mathbf{Q} \pm \overline{\mathbf{Q}}}$. These terms lead to a renormalization of the fourth order coefficient
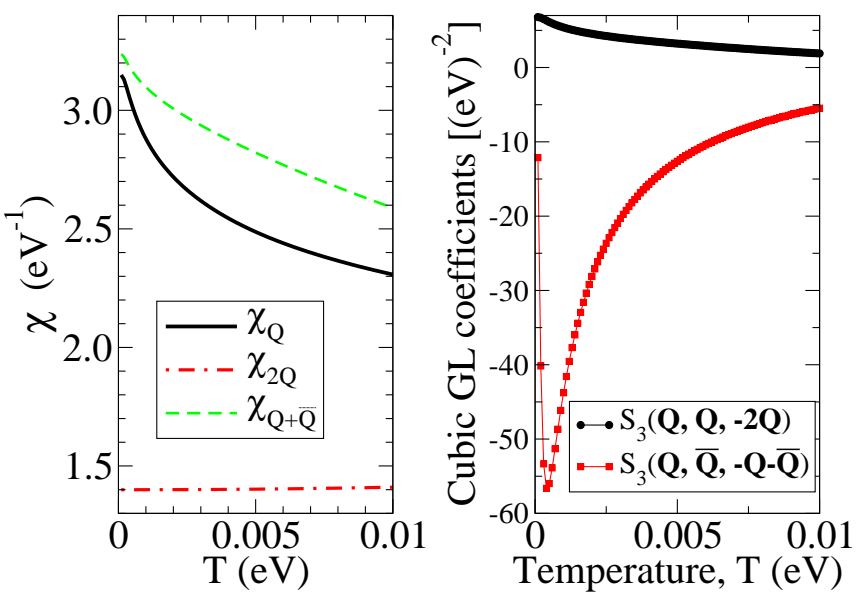

FIG. 7: (Color online) Left panel: Temperature dependence of the susceptibility at momenta $\mathbf{Q}, 2 \mathbf{Q}$, and $\mathbf{Q}+\overline{\mathbf{Q}}$ for momentum $\mathbf{Q} \approx(0.357 \pi / a, 0)$ (tb1 dispersion). Right panel: Temperature dependence of the third-order coefficients $S_{3}(\mathbf{Q}, \mathbf{Q},-2 \mathbf{Q})$ and $S_{3}(\mathbf{Q}, \overline{\mathbf{Q}},-\mathbf{Q}-\overline{\mathbf{Q}})$.

and, for a wide range of parameters, this can affect the preferred symmetry of the CDW, as shown in Ref. 20 for the case of the rare earth tri-tellurides. That is, inclusion of these harmonics leads to an additional contribution to $F\left(\Delta_{\mathbf{Q}}, \Delta_{\overline{\mathbf{Q}}}\right)$ of the form

$$
\begin{aligned}
& \delta F_{3}\left(\Delta_{\mathbf{Q}, \overline{\mathbf{Q}}}\right)= \\
& r_{2 \mathbf{Q}}\left(\left|\Delta_{2 \mathbf{Q}}\right|^{2}+\left|\Delta_{2} \overline{\mathbf{Q}}\right|^{2}\right)+r_{\mathbf{Q}+\overline{\mathbf{Q}}}\left(\left|\Delta_{\mathbf{Q}+\overline{\mathbf{Q}}}\right|^{2}+\left|\Delta_{\mathbf{Q}-\overline{\mathbf{Q}}}\right|^{2}\right) \\
& \quad+b_{\mathbf{Q}}\left(\Delta_{\mathbf{Q}}^{2} \Delta_{-2 \mathbf{Q}}+\Delta_{\overline{\mathbf{Q}}}^{2} \Delta_{-2} \overline{\mathbf{Q}}+c . c .\right) \\
& +c_{\mathbf{Q}}\left(\Delta_{-\mathbf{Q}-\overline{\mathbf{Q}}} \Delta_{\mathbf{Q}} \Delta_{\overline{\mathbf{Q}}}+\Delta_{\overline{\mathbf{Q}}-\mathbf{Q}} \Delta_{\mathbf{Q}} \Delta_{-\overline{\mathbf{Q}}}+c . c .\right)
\end{aligned}
$$

where

$$
\begin{aligned}
& b_{\mathbf{Q}}=-2 S_{3}(\mathbf{Q}, \mathbf{Q},-2 \mathbf{Q}) \\
& c_{\mathbf{Q}}=-4 S_{3}(\mathbf{Q}, \overline{\mathbf{Q}},-\mathbf{Q}-\overline{\mathbf{Q}})
\end{aligned}
$$

In the expression for $\delta F_{3}$ above, we omitted all terms higher than cubic order. These harmonics can be integrated out, as was shown by Yao et al $\stackrel{20}{ }$, and the resulting correction reads

$$
\delta F_{3}=-\frac{b_{\mathbf{Q}}^{2}}{r_{2 \mathbf{Q}}}\left(\left|\Delta_{\mathbf{Q}}\right|^{4}+\left|\Delta_{\overline{\mathbf{Q}}}\right|^{4}\right)-2 \frac{c_{\mathbf{Q}}^{2}}{r_{\mathbf{Q}+\overline{\mathbf{Q}}}}\left|\Delta_{\mathbf{Q}}\right|^{2}\left|\Delta_{\overline{\mathbf{Q}}}\right|^{2}
$$

These terms depend on the specific form of the interaction $g_{\mathbf{q}}$, which affects the coefficients $r_{\mathbf{Q}}$ and the choice of momentum Q. The coefficients $S_{3}$ are shown in Fig. 7.

One can estimate the effect of the corrections by notic- 

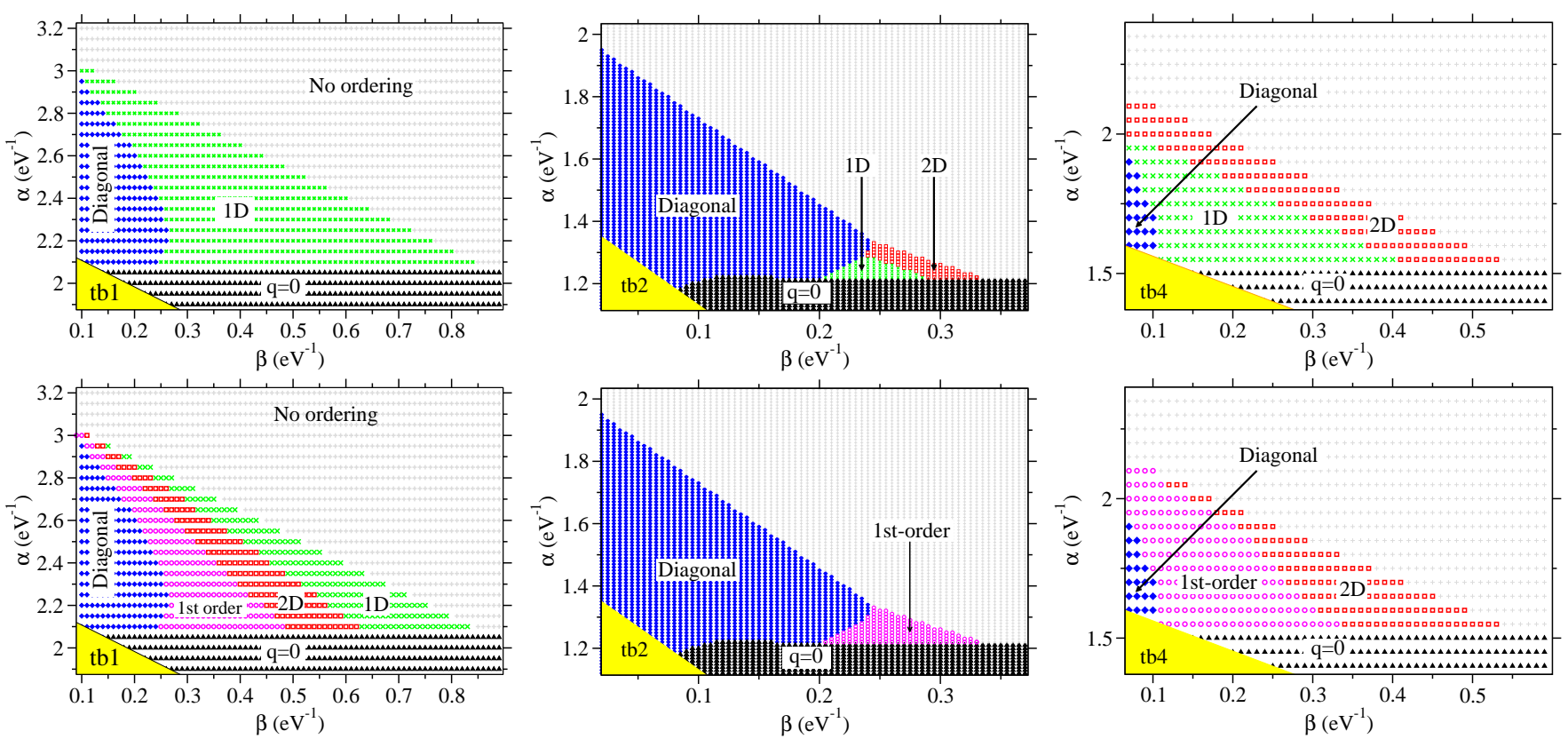

FIG. 8: (Color online) A refined version of the instability diagram of Fig. 5. The top (bottom) row corresponds to the instability diagrams without (with) cubic corrections. Instability at momenta lying along the Cu-O bonds can result in a 1D stripe phase (green crosses) or a 2D "checkerboard" phase (red squares). In the region marked by magenta circles, the cubic corrections that involve modes with momenta $\mathbf{Q}+\overline{\mathbf{Q}}$ destabilize the GL free energy (at the quartic level) by violating the stability condition $\gamma>-1$, corresponding to a first-order transition to a state with finite $\Delta_{\mathbf{Q}}, \Delta_{\overline{\mathbf{Q}}}, \Delta_{\mathbf{Q}+\overline{\mathbf{Q}}}$, and higher harmonics. The yellow shaded region (lower left corner) of each diagram indicates a regime where the transition temperature - formally obtained as a solution of Eq. (12) - is so high, the susceptibility peaks are smeared, and our simple GL approach should not be applied.

ing that the GL coefficients are modified as follows:

$$
\begin{aligned}
2 S_{A}+4 S_{B} & \rightarrow 2 S_{A}+4 S_{B}+\frac{4 S_{3}(\mathbf{Q}, \mathbf{Q},-2 \mathbf{Q})^{2}}{\frac{1}{2 g_{2} \mathbf{Q}}+\chi_{2} \mathbf{Q}} \\
8 S_{C}+4 S_{D} & \rightarrow 8 S_{C}+4 S_{D}+\frac{16 S_{3}(\mathbf{Q}, \overline{\mathbf{Q}},-\mathbf{Q}-\overline{\mathbf{Q}})^{2}}{\frac{1}{2 g_{\mathbf{Q}+\overline{\mathbf{Q}}}}+\chi_{\mathbf{Q}+\overline{\mathbf{Q}}}}
\end{aligned}
$$

In the situation shown in Fig. 4, the additional term in Eq. (19) can be neglected (i.e., the response at $2 \mathbf{Q}$ is typically small). On the other hand, the one in Eq. (20) can be significant, given the box-like structure of $\chi$ about the zone center (i.e., the response at $\mathbf{Q}+\overline{\mathbf{Q}}$ can be large). As a result, the net effect of the cubic corrections on the GL free energy is a reduction of the coefficient $\gamma$ describing the relative magnitude of the mixed term $\left|\Delta_{\mathbf{Q}}\right|^{2}\left|\Delta_{\overline{\mathbf{Q}}}\right|^{2}$. Consequently, the range of parameters for which the checkerboard symmetry is realized could be generally increased. Evaluation of the renormalized quartic coefficients from Eqs. (19) and (20) leads to a refined version of the instability diagram shown in Fig. 8

Dispersion tb1. In the region of the diagram that corresponds to low transition temperatures, the contribution from the quartic coefficients dominates over the thirdorder terms, and the preferable state is one-dimensional. For smaller curvature $\beta$, as one approaches the part of the diagram where the instability occurs along a diagonal wave vector, the contribution of the third-order terms becomes more pronounced and results in a transition to a two-dimensional state, as described above. Both the onedimensional and the two-dimensional cases are characterized by the dominant order parameter $\Delta_{\mathbf{Q}} \propto\left(T_{c}-T\right)^{1 / 2}$. In all cases, there are subdominant higher harmonics of the order parameter at wave vectors $2 \mathbf{Q}, \mathbf{Q}+\overline{\mathbf{Q}}$, etc. that in the vicinity of $T_{c}$ behave as integer powers of $\Delta_{\mathbf{Q}}$. For example, the most pronounced subdominant order parameter for the "checkerboard" state is $\Delta_{\mathbf{Q}+\overline{\mathbf{Q}}} \propto\left|\Delta_{\mathbf{Q}}\right|^{2} / r_{\mathbf{Q}+\overline{\mathbf{Q}}} \propto\left(T_{c}-T\right) / r_{\mathbf{Q}+\overline{\mathbf{Q}}}$. Note that as $\beta$ is reduced, $r_{\mathbf{Q}+\overline{\mathbf{Q}}}$ also decreases (Fig. 4 ). Thus for fixed $T_{c}-T$, the subdominant order $\Delta_{\mathbf{Q}+\overline{\mathbf{Q}}}$ will be growing, and one expects that the simple GL scenario considered so far will break down at some point.

Indeed, for even lower values of $\beta$, the renormalized value of $\gamma$ falls into the $\gamma<-1$ range. In other words, the third-order terms destabilize our reduced GL free energy expression that included only terms up to the fourth order in $\Delta_{\mathbf{Q}}$. This instability implies the necessity to include higher-order terms in the GL free energy expression for that part of the parameter space. These terms would generally restore the stability of the GL free energy, but would result in a first-order transition that cannot be described in any quantitative way using our approach. At the transition temperature $T_{c}$, a finite amplitude of $\Delta_{\mathbf{Q}}$ develops, and unless a weakly first order transition oc- 
curs, one in principle has to include an infinite number of terms in the GL free energy to describe it. The coupling of the order parameter $\Delta_{\mathbf{Q}}$ to the modes at $2 \mathbf{Q}$, $\mathbf{Q}+\overline{\mathbf{Q}}$, and higher harmonics in this case implies that $\Delta_{2 \mathbf{Q}}, \Delta_{\mathbf{Q}+\overline{\mathbf{Q}}}$, etc. also acquire finite values just below $T_{c}$. Since none of the higher harmonics have been observed in experiment, a detailed analysis of this phase is left for future study.

Dispersion tb2. The phase diagram for tb2 has significant differences from the tb1 case. The region of stability for diagonal ordering is enhanced at the expense of the $1 \mathrm{D}$ state, and in addition the $2 \mathrm{D}$ checkerboard state now appears. But the cubic corrections in this case are rather large, and with their inclusion, over the entire range of parameters where $1 \mathrm{D}$ and $2 \mathrm{D}$ second-order transitions would be expected, first-order transitions occur instead.

Dispersion tb4. The tb4 case looks more similar to the tb1 case than the tb2 one, except that $2 \mathrm{D}$ order is now present over a region of parameter space where order first appears. Inclusion of the cubic corrections leads to a complete suppression of the 1D state in favor of a first-order transition, but the checkerboard order remains stable.

\section{CONCLUSIONS}

One faces two difficulties when attempting to reconcile the atomic-scale modulations observed in real space by scanning tunneling microscopy (STM) with the energy dispersions obtained from photoemission. First, one must account for the fact that the modulation wave vector observed by STM is directed along the $\mathrm{Cu}-\mathrm{O}$ bonds, while the charge susceptibility extracted from the photoemission dispersions is largest along the diagonal of the zone. At the level of a weak-coupling theory with an effective electron-electron interaction, this apparent contradiction implies that the interaction must be momentum dependent. Moreover, one obtains rather stringent constraints on the range and strength of this effective interaction.

While both LSCO and Bi2212 have similar dispersions, the differing ordering tendencies observed in these materials should not come as a surprise, since small differences in the dispersions and the interaction coefficients $\alpha$ and $\beta$ are sufficient to move one from the $1 \mathrm{D}$ to $2 \mathrm{D}$ regions of the phase diagram. In all cases, one obtains a qualitatively similar instability diagram at the level of the susceptibility analysis, i.e., diagrams that determine whether the instability momentum lies along the $\mathrm{Cu}-\mathrm{O}$ bonds or diagonally.

Even when the interaction is such that the instability momentum is oriented correctly, there remains the question of a single $Q$ (stripe) versus a double $Q$ (checkerboard) state. This is where the results diverge sharply, even for materials with seemingly similar charge susceptibilities. As the quartic terms are sharply peaked functions of momentum near the "box" where the susceptibility is peaked, the detailed phase diagram that determines the energetically preferred state is a sensitive function of the dispersion.

Interestingly, the well known difficulty of differentiating whether the observed modulation patterns are unidirectional or checkerboard-like implies that although uni-directional behavior is the most likely, the real materials are near the $1 \mathrm{D}-2 \mathrm{D}$ phase boundary ${ }^{18}$ in Fig. 8 . It is also interesting to note that the cubic corrections tend to destabilize $1 \mathrm{D}$ order in favor of $2 \mathrm{D}$ order. In that context, recent observations in underdoped $\mathrm{YBa}_{2} \mathrm{Cu}_{3} \mathrm{O}_{6+x}$ have seen $2 \mathrm{D}$ order 26,27 and thus our work should be of interest in this regard.

\section{Acknowledgments}

We would like to thank S. Davis, O. Vafek and H. Yao for discussions and correspondence. This work was supported by the Materials Sciences and Engineering Division, Basic Energy Sciences, Office of Science, US DOE.
* Electronic address: norman@anl.gov

1 J. E. Hoffman, E. W. Hudson, K. M. Lang, V. Madhavan, H. Eisaki, S. Uchida and J. C. Davis, Science 295, 466 (2002).

2 J. E. Hoffman, K. McElroy, D.-H. Lee, K. M. Lang, H. Eisaki, S. Uchida and J. C. Davis, Science 297, 1148 (2002).

3 C. Howald, H. Eisaki, N. Kaneko, M. Greven and A. Kapitulnik, Phys. Rev. B 67, 014533 (2003).

4 M. Vershinin, S. Misra, S. Ono, Y. Abe, Y. Ando and A. Yazdani, Science 303, 1995 (2004).

5 Y. Kohsaka, C. Taylor, P. Wahl, A. Schmidt, J. Lee, K. Fujita, J. W. Alldredge, K. McElroy, J. Lee, H. Eisaki, S. Uchida, D.-H. Lee and J. C. Davis, Nature 454, 1072 (2008).
${ }^{6}$ C. V. Parker, P. Aynajian, E. H. daSilva Neto, A. Pushp, S. Ono, J. Wen, Z. Xu, G. Gu and A. Yazdani, Nature 468, 677 (2010).

7 T. Hanaguri, C. Lupien, Y. Kohsaka, D.-H. Lee, M. Azuma, M. Takano, H. Takagi and J. C. Davis, Nature 430, 1001 (2004).

${ }^{8}$ Y. Kohsaka, C. Taylor, K. Fujita, A. Schmidt, C. Lupien, T. Hanaguri, M. Azuma, M. Takano, H. Eisaki, H. Takagi, S. Uchida and J. C. Davis, Science 315, 1380 (2007).

9 H. D. Chen, J.-P. Hu, S. Capponi, E. Arrigoni and S.-C. Zhang, Phys. Rev. Lett. 89, 137004 (2002).

10 S.A. Kivelson, I. P. Bindloss, E. Fradkin, V. Oganesyan, J. M. Tranquada, A. Kapitulnik and C. Howald, Rev. Mod. Phys. 75, 1201 (2003).

11 H. D. Chen, O. Vafek, A. Yazdani and S.-C. Zhang, Phys. 
Rev. Lett. 93, 187002 (2004).

12 Z. Tešanović, Phys. Rev. Lett. 93, 217004 (2004).

13 C. Bena, S. Chakravarty, J. Hu and C. Nayak, Phys. Rev. B 69, 134517 (2004).

14 L. Balents, L. Bartosch, A. Burkov, S. Sachdev and K. Sengupta, Phys. Rev. B 71, 144508 (2005).

15 A. Melikyan and Z. Tešanović, Phys. Rev. B 71, 214511 (2005).

16 A. Ghosal, A. Kopp and S. Chakravarty, Phys. Rev. B 72, 220502 (2005).

17 T. Pereg-Barnea and M. Franz, Phys. Rev. B 74, 014518 (2006).

18 J. A. Robertson, S. A. Kivelson, E. Fradkin, A. C. Fang and A. Kapitulnik, Phys. Rev. B 74, 134507 (2006).

${ }^{19}$ K. Seo, H.-D. Chen and J. Hu, Phys. Rev. B 76, 020511 (2007).

20 H. Yao, J. A. Robertson, E.-A. Kim and S. A. Kivelson, Phys. Rev. B 74, 245126 (2006).

21 M. R. Norman, Phys. Rev. B 75, 184514 (2007).

22 M. R. Norman, M. Randeria, H. Ding and J. C. Campuzano, Phys. Rev. B 52, 615 (1995).
23 A. Kaminski, H. M. Fretwell, M. R. Norman, M. Randeria, S. Rosenkranz, U. Chatterjee, J. C. Campuzano, J. Mesot, T. Sato, T. Takahashi, T. Terashima, M. Takano, K. Kadowaki, Z. Z. Li and H. Raffy, Phys. Rev. B 71, 014517 (2005).

24 X. J. Zhou, T. Yoshida, D.-H. Lee, W. L. Yang, V. Brouet, F. Zhou, W. X. Ti, J. W. Xiong, Z. X. Zhao, T. Sasagawa, T. Kakeshita, H. Eisaki, S. Uchida, A. Fujimori, Z. Hussain and Z.-X. Shen, Phys. Rev. Lett. 92, 187001 (2004).

${ }^{25}$ H. J. Schulz, Phys. Rev. Lett 64, 1445 (1990).

${ }^{26}$ G. Ghiringhelli, M. Le Tacon, M. Minola, S. BlancoCanosa, C. Mazzoli, N. B. Brookes, G. M. De Luca, A. Frano, D. G. Hawthorn, F. He, T. Loew, M. Moretti Sala, D. C. Peets, M. Salluzzo, E. Schierle, R. Sutarto, G. A. Sawatzky, E. Weschke, B. Keimer and L. Braicovich, Science 337, 821 (2012).

27 J. Chang, E. Blackburn, A. T. Holmes, N. B. Christensen, J. Larsen, J. Mesot, R. Liang, D. A. Bonn, W. N. Hardy, A. Watenphul, M. v. Zimmermann, E. M. Forgan and S. M. Hayden, Nature Phys. 8, 871 (2012). 